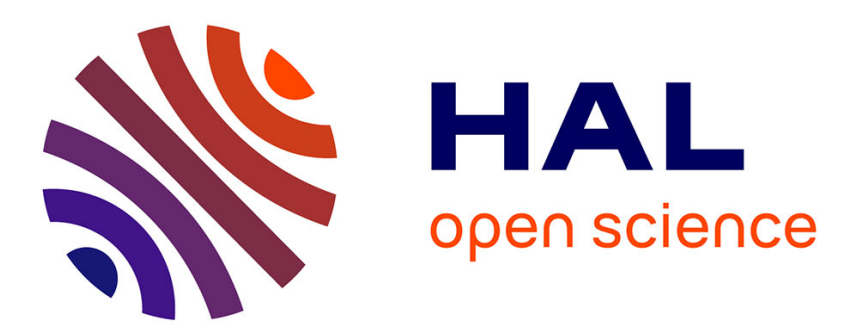

\title{
Photonic bandgaps of periodic multilayers with diffuse interfaces
}

\author{
Jean-Michel André, Philippe Jonnard
}

\section{To cite this version:}

Jean-Michel André, Philippe Jonnard. Photonic bandgaps of periodic multilayers with diffuse interfaces. Journal of the Optical Society of America B, 2013, 30 (8), pp.2296-2300. 10.1364/JOSAB.30.002296 . hal-00854489

\section{HAL Id: hal-00854489 \\ https://hal.science/hal-00854489}

Submitted on 27 Aug 2013

HAL is a multi-disciplinary open access archive for the deposit and dissemination of scientific research documents, whether they are published or not. The documents may come from teaching and research institutions in France or abroad, or from public or private research centers.
L'archive ouverte pluridisciplinaire HAL, est destinée au dépôt et à la diffusion de documents scientifiques de niveau recherche, publiés ou non, émanant des établissements d'enseignement et de recherche français ou étrangers, des laboratoires publics ou privés. 


\title{
Photonic bandgaps of periodic multilayers
}

\section{with diffuse interfaces}

\author{
Jean-Michel André*, Philippe Jonnard \\ Laboratoire de Chimie Physique - Matière et Rayonnement, CNRS UMR 7614, Université Pierre et Marie Curie, 11 rue Pierre et Marie Curie, \\ 75231 Paris CEDEX 05, France \\ *Corresponding author: jean-michel.andre1@upmc.fr
}

\begin{abstract}
The photonic bandgap of periodic multilayers with diffuse interface is calculated by considering an empirical model for the interdiffusion profile. The model for the diffuse profile is based on the error function $\operatorname{Erf}$ and a characteristic parameter $\sigma$. The model is valid for multilayer structure with arbitrary layer thickness. It is shown that the width of the bandgap varies with the value of $\sigma$ and the Brillouin zone boundary. Numerical examples are presented. It is suggested that measuring the ratio of the width of the bandgaps at different orders, that is to say determining experimentally the ratio of width of the different Bragg peaks on a reflectivity curve, can allow estimating the thickness of the interdiffusion layer.
\end{abstract}

This paper was published in JOSA B and is made available as an electronic reprint with the permission of OSA. The paper can be found at the following URL on the OSA website:

http://www.opticsinfobase.org/josab/abstract.cfm?uri=josab-30-8-2296. Systematic or multiple reproduction or distribution to multiple locations via electronic or other means is prohibited and is subject to penalties under law. 


\title{
Photonic bandgaps of periodic multilayers with diffuse interfaces
}

\author{
Jean-Michel André* and Philippe Jonnard \\ Laboratoire de Chimie Physique-Matière et Rayonnement, CNRS UMR 7614, Université Pierre et Marie Curie, \\ 11 rue Pierre et Marie Curie, 75231 Paris CEDEX 05, France \\ ${ }^{*}$ Corresponding author: jean-michel.andre1@upmc.fr
}

Received April 26, 2013; revised July 12, 2013; accepted July 12, 2013; posted July 12, 2013 (Doc. ID 189578); published July 29, 2013

\begin{abstract}
The photonic bandgap of periodic multilayers with diffuse interfaces is calculated by considering an empirical model for the interdiffusion profile. The model for the diffuse profile is based on the error function $\operatorname{Erf}$ and a characteristic parameter $\sigma$. The model is valid for multilayer structures with an arbitrary layer thickness. It is shown that the width of the bandgap varies with the value of $\sigma$ and the Brillouin zone boundary. Numerical examples are presented. It is suggested that measuring the ratio of the width of the bandgaps at different orders, that is, determining experimentally the ratio of the widths of the different Bragg peaks on a reflectivity curve, can allow estimation of the thickness of the interdiffusion layer. (c) 2013 Optical Society of America

OCIS codes: $\quad$ (340.0340) X-ray optics; (340.7480) X-rays, soft x-rays, extreme ultraviolet (EUV); (160.5293)

Photonic bandgap materials; (230.4170) Multilayers; (350.4238) Nanophotonics and photonic crystals.

http://dx.doi.org/10.1364/JOSAB.30.002296
\end{abstract}

\section{INTRODUCTION}

Periodic multilayer structures used in optics can be regarded as one-dimensional photonic crystals. It is well known that this kind of structure presents photonic bandgaps that are frequency domains where no propagating mode is allowed [1]. For ideal structures with sharp and flat interfaces, the calculation of the locations and widths of the forbidden bandgaps is standard [1-3]. However, generally the interfaces between the layers are not ideal but present roughness or interdiffusion or both. It has been recognized that these imperfections can affect the width or even the occurrence of the bandgaps [4,5]. In [5], the authors consider the propagation of waves (not necessarily electromagnetic waves but spin, elastic, and other waves) in a multilayer structure with a finite thickness of the interfaces; the interfaces are modeled by a Jacobian elliptic sine function, and it is shown that in this case, the dependence of the widths of the bandgaps can differ significantly for the different Brillouin zones.

Nevertheless the model of [5] does not deal with the structure of arbitrary layer thicknesses. One-dimensional photonic crystals of nanometric scale are now largely implemented as monochromators in soft x-ray optics [6], a fact that has largely inspired the present work. Note that the introduction of interdiffusion in perfect multilayer structures (with step-like interfaces) leads to a structure where the dielectric constant is varied continuously as in the so-called rugate filters [7]. This means that one could envisage implementing the theory given in this paper to the study of a rugate filter with a periodic profile.

In the present work, we consider the case of an electromagnetic wave in a bilayered periodic structure with an arbitrary ratio of layer thicknesses, despite which we could consider applying a generalization of this work to a rugate filter. The interfaces are diffuse and considered as transition layers, the shape of which is described by a function (error function) validated by a lot of experimental data. In Section 2 , we give the theory required to determine the dispersion curves and subsequently the widths of the bandgaps; in Section 3, we present the model for the diffuse interfaces. The Section $\underline{4}$ gives the expression of the widths of the bandgaps in the two-wave approximation, and in Section 5, we present numerical examples.

\section{DISPERSION CURVE IN A PERIODIC MULTILAYER}

We consider the propagation of a harmonic electromagnetic wave of frequency $\omega$ with a wave vector $\mathbf{k}$, within a periodic multilayer structure. The $z$ axis is the direction of stratification, and the waves travel in the $y-z$ plane, as shown in Fig. 1 . The wave vector $\mathbf{k}$ can be decomposed into an invariant tangential component $\boldsymbol{k}_{\|}$lying in the $x-y$ plane and a normal component $\boldsymbol{k}_{\perp}$ along the $z$ axis.

According to Snell's law, one has

$$
k_{\|}^{2}+k_{\perp}^{2}=k^{2}=\varepsilon(z) k_{0}^{2},
$$

where $\varepsilon(z)$ is the dielectric constant at the location $z$ and $k_{0}=$ $\omega / c$ is the wavenumber in vacuum, $c$ being the light speed in vacuum. As usual, the field can be decomposed into a transverse electric (TE) field, for which the electric field $E$ is only one nonvanishing component $E_{x}=U(z) \exp \left(i k_{\|} y\right)$, and a transverse magnetic (TM) field, for which the magnetic field $H$ is only one nonvanishing component $H_{x}=U(z) \exp \left(i k_{\|} y\right)$. In both cases, the amplitude $U(z)$ obeys the following differential equation:

$$
\frac{d^{2} F(z)}{d z^{2}}+h^{2}(z) F(z)=0
$$




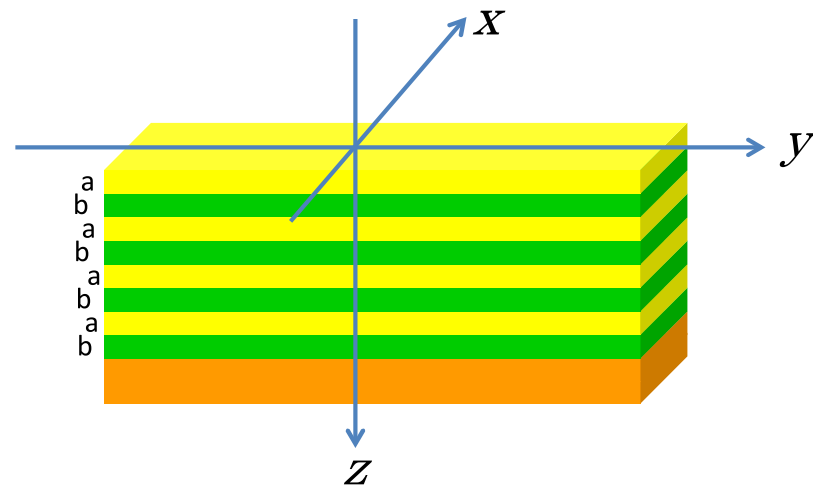

Fig. 1. Scheme of the bilayer system and geometry used. The period of the multilayer $D$ is the sum of the thicknesses of the $a$ and $b$ layers. The $\gamma$-ratio is the ratio of the thickness of the $a$ layer to the period.

where $h^{2}(z)=k_{\perp}^{2}(z)=\varepsilon(z) k_{0}^{2}-k_{\|}^{2}$ and $F(z)=U(z)$ for the TE polarization case and $h^{2}(z)=k_{\perp}^{2}(z)-\left[\left(d^{2} \operatorname{Ln}(\sqrt{\varepsilon(z)}) / d z^{2}\right)+\right.$ $\left.(d \operatorname{Ln}(\sqrt{\varepsilon(z)}) / d z)^{2}\right]$ and $F(z)=U(z) / \sqrt{\varepsilon(z)}$ for the TM polarization case [8]. Since the dielectric function $\varepsilon(z)$ is a periodic function of the depth $z$, Eq. (2) is a Hill equation [9,10], the solution of which is given by the Bloch-Floquet theorem: there is a wavenumber $K$ (Bloch wavenumber) and a periodic function $u(z)$ such that

$$
U(z)=\exp (i K z) u(z)
$$

The Bloch wavenumber satisfies the dispersion formula [10], but its value is not relevant in the following calculation of the bandgap width. The periodic function can be expanded in a Fourier series so that the amplitude becomes

$$
U(z)=\exp (i K z) \sum_{n=-\infty}^{\infty} u_{n} \exp (i n G z)
$$

Taking into account the periodicity of $\varepsilon(z)$, this quantity can also be expanded in a Fourier series:

$$
\varepsilon(z)=\sum_{p=-\infty}^{\infty} \varepsilon_{p} \exp (i p G z)
$$

where $G$ is the reciprocal distance. Substituting Eqs. (4) and (5) in Eq. (2), one gets the following equation in the TE case:

$$
\begin{aligned}
& \sum_{n=-\infty}^{n=\infty} \exp (i n G z)\left[\varepsilon_{0} k_{0}^{2}-\left((K-n G)^{2}+k_{\|}^{2}\right)\right] u_{n} \\
& \quad=k_{0}^{2}\left(\sum_{p=-\infty}^{+\infty} \epsilon_{p} \exp (i p G z)\right)\left(\sum_{n=-\infty}^{+\infty} u_{n} \exp (i n G z)\right)
\end{aligned}
$$

Since each Fourier component must have the same coefficient in the two members of this equation, it becomes

$$
\left[\varepsilon_{0} k_{0}^{2}-\left((K-n G)^{2}+k_{\|}^{2}\right)\right] u_{n}=k_{0}^{2}\left(\sum_{t=-\infty}^{+\infty} u_{t} \epsilon_{n-t}\right)
$$

Expressing $u_{n}$ from this equation and reintroducing it into Eq. (7), one obtains

$$
\begin{aligned}
& {\left[\varepsilon_{0} k_{0}^{2}-\left((K-n G)^{2}+k_{\|}^{2}\right)\right] u_{n}} \\
& \quad=k_{0}^{4} \sum_{t=-\infty}^{+\infty} \sum_{p=-\infty}^{+\infty} u_{p} \frac{\epsilon_{t-p} \epsilon_{n-t}}{\left[\varepsilon_{0} k^{2}-\left((K-t G)^{2}+k_{\|}^{2}\right)\right]} .
\end{aligned}
$$

At the first order of the perturbation theory $(p=n)$, the main branch of the dispersion curve $(p=0)$ is given by the formula

$$
\left[\varepsilon_{0} k_{0}^{2}-\left(K^{2}+k_{\|}^{2}\right)\right]=k_{0}^{4} \sum_{t=-\infty}^{+\infty} \frac{\epsilon_{t} \epsilon_{-t}}{\left[\varepsilon_{0} k^{2}-\left((K-t G)^{2}+k_{\|}^{2}\right)\right]} .
$$

Provided $\Delta \varepsilon \ll \varepsilon_{a}, \varepsilon_{b}$, where $\Delta \varepsilon=\left|\varepsilon_{b}-\varepsilon_{a}\right|$ and $\varepsilon_{a}\left(\varepsilon_{b}\right)$ is the dielectric constant of the material constituting the layer $a(b)$ of the multilayer, the condition for the two-wave approximation is fulfilled. Under this condition, if one selects from the sum on the right-hand side of Eq. (12) the term resonant in the vicinity of the $t$ th Brillouin zone boundary, the dispersion law reads

$$
\left[\varepsilon_{0} k_{0}^{2}-\left(K^{2}+k_{\|}^{2}\right)\right]\left[\varepsilon_{0} k_{0}^{2}-\left((K-t G)^{2}+k_{\|}^{2}\right)\right]=k_{0}^{4} \epsilon_{t} \epsilon_{-t} .
$$

For the TM case, the calculation is formally the same as the one for the TE case, provided that one replaces the original dielectric constant $\varepsilon(z)$ with the modified one $\tilde{\varepsilon}(z)$ related to $\varepsilon(z)$ by the relationship

$$
\tilde{\varepsilon}(z)=\varepsilon(z)+\frac{1}{2 \varepsilon(z) k_{0}^{2}} \frac{d^{2} \varepsilon(z)}{d z^{2}}-\frac{3}{4 \varepsilon(z)^{2} k_{0}^{2}}\left(\frac{d \varepsilon(z)}{d z}\right)^{2} .
$$

This formula is deduced from the expression of $h^{2}(z)$ in the TM case. The problem of the validity of the two-wave approximation is beyond the scope of this paper; it has been considered in [11].

\section{MODEL FOR THE IN-DEPTH PROFILE}

The purpose of this section is to give a plausible model to describe the in-depth profile of the periodic structure with diffuse interfaces. We consider a bilayered system with period $D$ and a duty cycle (so-called gamma ratio) $\gamma$ (see Fig. 1); the thickness of the first layer $a$ is $\gamma D$ and the thickness of the other layer $b$ is $(1-\gamma) D$. The interfaces are not supposed to be sharp but diffuse, forming a transition layer characterized by a parameter $\sigma$. A lot of experimental data indicate that a large class of diffuse interfaces can be modeled by the cumulative distribution function for the normal distribution $\tilde{\rho}(z, \sigma)[\underline{12}, \underline{13}]$ :

$$
\tilde{\rho}(z, \sigma)=\frac{1}{2}\left[1+\operatorname{Erf}\left(\frac{z}{\sqrt{2} \sigma}\right)\right] .
$$

In our approach, the parameter $\sigma$ is a quantity that can be regarded as the characteristic thickness of an interdiffusion layer; interdiffusion can be viewed as a one-dimensional inhomogeneity. Then, in principle, $\sigma$ cannot describe the geometrical roughness (GR) at the interface of two layers, since GR is a two-dimensional inhomogeneity that can give rise to light scattering (nonspecular reflection). Nevertheless, it is typical to account for the GR by a transition layer similar to an interdiffusion layer modeled by Eq. (12); the thickness of the 
transition layer is then equal to $2 \sqrt{2} \sigma$ [6]. At this stage, it is convenient to introduce the quantity $\delta$ as the ratio $\sigma / D$ and the correponding distribution $\rho(z, \delta)$ deduced from $\tilde{\rho}(z, \sigma)$ by replacing $\sigma$ by $\delta$. In the framework of this model, the in-depth distribution of the dielectric function $\varepsilon(z, \delta)$ can be written as follows:

$$
\varepsilon(z, \delta)=\varepsilon_{a}+\Delta \varepsilon \Omega(z, \delta)
$$

with

$$
\begin{aligned}
\Omega(z, \delta)= & \frac{\lim }{N \rightarrow \infty}\left(\sum _ { n = - N } ^ { + N } \left(\rho\left(+n D+\frac{\gamma D}{2}+z, \delta\right)\right.\right. \\
& \left.\left.+\rho\left(-n D+\frac{\gamma D}{2}-z, \delta\right)\right)-(2 N+1)\right) .
\end{aligned}
$$

The function $\Omega(z, \delta)$ can be expanded in a Fourier series, so that

$$
\varepsilon(z)=\varepsilon_{a}+\Delta \varepsilon \sum_{p=-\infty}^{\infty} \Omega_{p} \exp (-i p G z)
$$

where $\Omega_{p}(\delta)$ are the coefficients in the Fourier series of $\Omega(z, \delta)$, which are given by

$$
\begin{aligned}
\Omega_{p}(\delta)= & \frac{i}{4 p \pi} e^{-\left(2 p^{2} \pi^{2} \delta^{2}\right)}\left[e ^ { - i p \pi \gamma } \left(\operatorname{Erf}\left(\frac{(1+\gamma) / \delta-4 i p \pi \delta^{2}}{2 \sqrt{2}}\right)\right.\right. \\
& \left.+\operatorname{Erf}\left(\frac{(1-\gamma) / \delta+4 i p \pi \delta^{2}}{2 \sqrt{2}}\right)\right) \\
& +e^{i p \pi \gamma}\left(\operatorname{Erf}\left(\frac{(-1+\gamma) / \delta+4 i p \pi \delta^{2}}{2 \sqrt{2}}\right)\right. \\
& \left.\left.-\operatorname{Erf}\left(\frac{(1+\gamma) / \delta+4 i p \pi \delta^{2}}{2 \sqrt{2}}\right)\right)\right]
\end{aligned}
$$

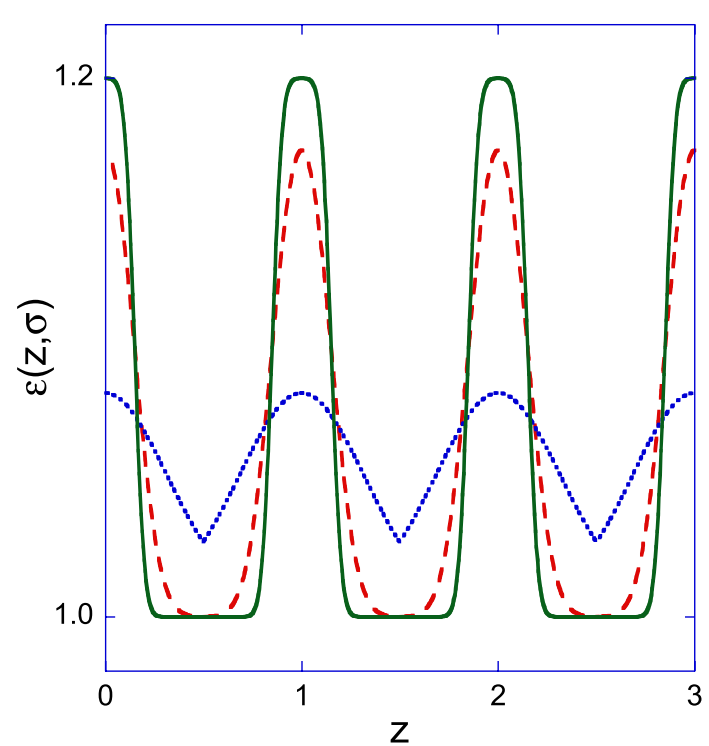

Fig. 2. In-depth profile of the dielectric function $\varepsilon(z, \sigma)$ for the following parameters: $D=1, \gamma=0.4, \delta=0.04$ (solid line), 0.1 (dashed line), and 0.3 (dotted line), $\varepsilon_{a}=1.0$, and $\Delta \varepsilon=0.2$, computed from the Fourier series by means of Eqs. (15) and (16).
It can be verified that $\Omega_{p}(\delta=0)=\sin (p \pi \gamma) /(p \pi)$, in agreement with the standard results for ideal multilayers (i.e., with sharp interfaces) [1-ㄱ]

Figure 2 shows the in-depth profile of the dielectric function $\varepsilon(z, \bar{\delta})$ for the following parameters: $D=1, \gamma=0.4$, $\delta=0.04,0.1,0.3, \varepsilon_{a}=1.0$, and $\Delta \varepsilon=0.2$, computed from the Fourier series Eqs. (15) and (16). Exactly the same profiles are obtained from the direct computation using Eqs. (13) and (14). This result tends to prove that Eq. (16) remains relevant even when ratio $\delta / \gamma$ becomes close to unity, and then that our approach remains valid even when the lamellar-like profile disappears and becomes more sine-like. Of course further investigations (both theoretical and experimental) would be necessary to validate this assertion. In this case one could envisage treating some class of rugate filters with the present method.

\section{PHOTONIC BANDGAPS IN THE TWO- WAVE APPROXIMATION}

To obtain the dispersion curve $\omega=\omega(K)$, one has to insert Eqs. (15) and (16) into Eq. (10); this equation will provide the shape of $\omega(\bar{K})$, in the vicinity of the Brillouin zone boundaries for which the value of the Bloch wavenumber takes the values

$$
K=p \frac{G}{2}
$$

Hence, in the two-wave approximation, one has at the boundary of the $p$ th Brillouin zone:

$$
\left[\varepsilon_{0} \frac{\omega_{2}}{c^{2}}-\left(\frac{p^{2} G^{2}}{4}+k_{\|}^{2}\right)\right]^{2}=(\Delta \varepsilon)^{2} \frac{\omega^{4}}{c^{4}}\left(\Omega_{p}(\delta)\right)^{2},
$$

since

$$
\Omega_{-p}(\delta)=\Omega_{p}(\delta)
$$

From Eq. (18), it is seen (see Appendix A) that the width $\Delta \omega_{p}$, of the $p$ th bandgap, which is at the $p$ th Brillouin zone boundary, is given by

$$
\Delta \omega_{p}=\frac{\Delta \varepsilon}{\varepsilon_{0}} \omega \Omega_{p}(\delta)
$$

It follows from Eq. (20) that the bandgap width depends on the characteristic parameter $\delta$ of the interdiffusion layer and on the order of the bandgap through the term $\Omega_{p}(\delta)$. In the next section we illustrate this phenomenon with some numerical examples. Finally, let us note that the ratios $\left(\Delta \omega_{p}\right) /\left(\Delta \omega_{q}\right)$ do not depend on $(\Delta \varepsilon) /\left(\varepsilon_{0}\right)$, provided dispersion is not taken into account.

\section{NUMERICAL EXAMPLES}

Figure 3 displays the ratios $\left(\Delta \omega_{2}\right) /\left(\Delta \omega_{1}\right)$ and $\left(\Delta \omega_{3}\right) /\left(\Delta \omega_{1}\right)$ for the following parameters: $D=1$ and $\gamma=0.3$ versus the parameter $\delta$ for the TE case, assuming dispersionless media. We observe that the ratios decrease when the value of $\delta$ increases, in agreement with the results obtained with the model of [5]. We have computed these ratios using the transfermatrix method [2,8] for $\delta=0$; for $\left(\Delta \omega_{2}\right) /\left(\Delta \omega_{1}\right)($ at $\delta=0)$, 


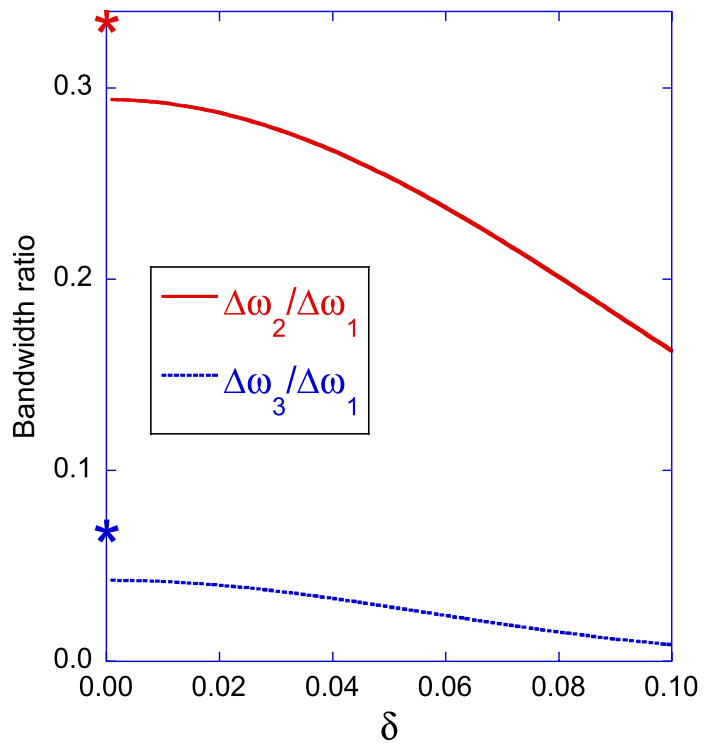

Fig. 3. Ratios of the widths of the bandgap at different Brillouin zone boundaries calculated with $D=1$ and $\gamma=0.3$ for the TE case. The stars give the values obtained from a computation using the transfer-matrix method.

we find 0.335 and for $\left(\Delta \omega_{3}\right) /\left(\Delta \omega_{1}\right)($ at $\delta=0)$, we get 0.067 . These values can be compared to the values given from Eq. (20), which are 0.294 and 0.042 , respectively (see Fig. 3). These discrepancies (12\% for $\left(\Delta \omega_{2}\right) /\left(\Delta \omega_{1}\right)$ and $37 \%$ for $\left.\left(\Delta \omega_{3}\right) /\left(\Delta \omega_{1}\right)\right)$ can be attributed to the two-wave approximation, since the condition $\Delta \varepsilon \ll \varepsilon_{a}, \varepsilon_{b}$ is not really satisfied in our example: $\Delta \varepsilon=0.2$ and $\varepsilon_{a}=1$. The discrepancy falls down to $7 \%$ (for $\left(\Delta \omega_{2}\right) /\left(\Delta \omega_{1}\right)$ ) and $32 \%$ (for $\left(\Delta \omega_{3}\right) /\left(\Delta \omega_{1}\right)$ ). When $\Delta \varepsilon=0.05$. This result is in agreement with the fact that the validity of the two-wave approximation increases when the ratio $\Delta \varepsilon / \varepsilon_{a}$ decreases [11].

\section{CONCLUSION AND PERSPECTIVES}

Our theory allows the bandgap widths to be determined for periodic multilayers with diffuse interfaces and arbitrary layer thicknesses. The empirical model used to describe the interdiffusion profile is in agreement with a great number of experimental results, which greatly validates of our approach.

This theory shows that it could be possible to find the parameter $\sigma$ of a diffuse interface, that is, to determine the effective thickness of the interfacial transition layer, by measuring the ratio between the widths of the bandgaps at the boundaries of several Brillouin zones. Experimentally, this means that measuring the ratio of the widths of the Bragg peaks on a reflectivity curve should allow the determination of the width of the transition layer in a multilayer where interdiffusion is present. Provided that the conditions of validity of the two-wave approximation are fullfilled, this operation can be done by means of Eq. (20); otherwise tranfer-matrix approach can be implemented. However, the present model is developed without considering absorption. Let us also note that the instrumental broadening is not taken into account. So, in order to compare with the present model, experimental data should be obtained in the hard x-ray range where absorption is minimal and with a reflectometer with good angular resolution. The scattering effects arising from interface roughness are not considered in this paper; as outlined in Section 3, the transition layer is assumed to come from interdiffusion, not from lateral inhomogeneities. Moreover, the separation of the effect of roughness with that of interdiffusion on the width of the bandgap must be carefully considered; this problem is not a simple task.

The model can be extended without difficulty to systems with asymmetric interfaces that have been often observed; see $[14,15]$ for example. Nevertheless, in the case of an asymmetric transition layer, the inverse problem consisting in finding the two parameters $\sigma_{a / b}$ and $\sigma_{b / a}$ from the measurements of the ratios of the bandgap widths becomes a more difficult task. The extension to the complex values of the dielectric constant is also of interest in treating the case of multilayers in the spectral regions where absorption becomes important, as in the extreme UV ranges.

\section{APPENDIX A}

This appendix gives the proof of Eq. (20). From Eq. (18),

$$
\varepsilon_{0} \nu_{ \pm}-\beta= \pm \alpha \nu_{ \pm}
$$

where

$$
\begin{aligned}
\nu_{ \pm} & =\left(\frac{\omega_{ \pm}}{c}\right)^{2}, \\
\alpha & =\Delta \varepsilon \Omega_{p}(\delta), \\
\beta & =\left(\frac{p^{2} G^{2}}{4}+k_{\|}^{2}\right) .
\end{aligned}
$$

It follows that

$$
\varepsilon_{0}\left(\nu_{+}-\nu_{-}\right)=\alpha\left(\nu_{+}+\nu_{-}\right)
$$

or

$$
\omega_{+}-\omega_{-}=\frac{\alpha}{\varepsilon_{0}}\left(\frac{\omega_{+}^{2}+\omega_{-}^{2}}{\omega_{+}+\omega_{-}}\right)
$$

With

$$
\omega_{ \pm}=\omega_{0} \pm \frac{1}{2} \Delta \omega
$$

we obtain

$$
\left(\omega_{+}-\omega_{-}\right)=\Delta \omega_{0}=\frac{\alpha}{\varepsilon_{0}} \frac{2 \omega_{0}^{2}+\frac{1}{2} \Delta \omega^{2}}{2 \omega_{0}},
$$

that is,

$$
\Delta \omega_{0} \approx\left(\frac{\Delta \varepsilon}{\varepsilon_{0}}\right) \omega_{0} \Omega_{p}(\delta)
$$

\section{REFERENCES}

1. J. D. Joannopoulos, S. G. Johnson, J. N. Winn, and R. D. Meade, Photonic Crystals: Molding the Flow of Light, 2nd ed. (Princeton University, 2008).

2. P. Yeh, Optical Waves in Layered Media (Wiley, 2005).

3. S. L. Chuang, Physics of Photonic Devices, 2nd ed. (Wiley, 2009). 
4. V. A. Ignatchenko, Y. I. Mankov, and A. A. Maradudin, "The spectrum and damping of waves in partially randomized multilayers," J. Phys. Condens. Matter 11, 2773-2790 (1999).

5. V. A. Ignatchenko, Y. I. Mankov, and A. A. Maradudin, "Wave spectrum of multilayers with finite thicknesses of interfaces," Phys. Rev. B 62, 2181-2184 (2000).

6. D. Attwood, Soft X-Rays and Extreme Ultraviolet Radiation (Cambridge University, 2000).

7. B. G. Bovard, "Rugate filter theory: an overview," Appl. Opt. 32, 5427-5442 (1993)

8. B. Pardo, T. Megademini, and J.-M. André, "X-UV synthetic interference mirrors: theoretical approach," Rev. Phys. Appl. 23, 1579-1597 (1988).

9. G. W. Hill, "On the part of the motion of the lunar perigee which is a function of the mean motions of the sun and moon," Acta Math. 8, 1-36 (1886).

10. J.-M. André and B. Pardo, "Onde électromagnétique sinusoidale dans un milieu périodiquement stratifié," Opt. Commun. 66, 249-254 (1988).
11. O. Francescangeli and A. Morini, "On the limits of validity of the two-wave approximation in the dynamical theory of electromagnetic scattering by periodic dielectric media," J. Phys. I 6, 705-723 (1996).

12. C. Fermon, F. Ott, and A. Menelle, "Neutron reflectometry," in X-ray and Neutron Reflectivity, J. Daillant and A. Gibaud, eds., Lecture Notes in Physics No. 770 (Springer, 2009), pp. 183-234.

13. P. Shewmon, Diffusion in Solids, 2nd ed. (Shewmon, 2010).

14. H. Maury, P. Jonnard, J.-M. André, J. Gautier, F. Bridou, F. Delmotte, and M.-F. Ravet, "Interface characteristics of $\mathrm{Mo} / \mathrm{Si}$ and $\mathrm{B} 4 \mathrm{C} / \mathrm{Mo} / \mathrm{Si}$ multilayers using nondestructive X-ray techniques," Surf. Sci. 601, 2315-2322 (2007).

15. H. Maury, J.-M. André, K. Le Guen, N. Mahne, A. Giglia, S. Nannarone, F. Bridou, F. Delmotte, and P. Jonnard, "Analysis of periodic Mo/Si multilayers: influence of the Mo thickness," Surf. Sci. 603, 407-411 (2009). 